\title{
Investigating the Interaction of Nanodiamonds with Human Serum Albumin and Induced Cytotoxicity
}

\author{
Miaomiao Chen, ${ }^{1,2}$ Xiaoshuang Zuo, ${ }^{1}$ Qinqin Xu, ${ }^{1}$ Rong Wang, ${ }^{2}$ Suhua Fan, \\ and Hai $\mathrm{Wu} \mathbb{D i D}^{1,2}$ \\ ${ }^{1}$ School of Chemistry and Materials Engineering, Fuyang Normal University, Fuyang, Anhui 236037, China \\ ${ }^{2}$ Anhui Province Key Laboratory of Environmental Hormone and Reproduction, \\ Anhui Provincial Key Laboratory for Degradation and Monitoring of Pollution of the Environment, Fuyang \\ Normal University, Fuyang, Anhui 236037, China
}

Correspondence should be addressed to Hai Wu; wuhai317@126.com

Received 22 May 2019; Accepted 26 August 2019; Published 19 September 2019

Academic Editor: Jeongkwon Kim

Copyright (c) 2019 Miaomiao Chen et al. This is an open access article distributed under the Creative Commons Attribution License, which permits unrestricted use, distribution, and reproduction in any medium, provided the original work is properly cited.

\begin{abstract}
Nanodiamonds (NDs) have been recognized as emerging carbon-based delivery vehicles due to their biocompatibility. NDs were reported to be nontoxic and suited for biomedical applications in the complete cell culture medium. However, in this study, the cytotoxicity of NDs in serum-free medium was studied and indicated that serum proteins in cell culture medium played significant effect on the toxicity of NDs. Therefore, the interaction mechanism between NDs and a serum protein (human serum albumin, HSA) was first investigated by fluorescence quenching technique and circular dichroism (CD) spectrometry. The results suggest that HSA strongly bonds on the NDs surface to form "protein corona," which not only prevents the aggregation of NDs and improves its stability but also inhibits the cytotoxicity of NDs. In serum-free medium, NDs exhibited obvious toxicity toward the human lung epithelial cell line (BEAS-2B) and showed concentration-dependent cytotoxicity. In the presence of bovine serum albumin (BSA), which shares structural homology and similar properties with HSA, toxicity of NDs was apparently inhibited. Therefore, the interaction between serum protein and NDs should be considered in the understanding of the biological effects of NDs exposure in biomedical applications.
\end{abstract}

\section{Introduction}

Carbon-based nanomaterials have been paid more interests in the fields of biotechnological and biomedical applications, including fullerenes, carbon nanotubes, graphene, and nanodiamonds (NDs), as well as their functionalized forms $[1,2]$. Previous reports have demonstrated that NDs are the least toxic of all carbon-based nanomaterials studied so far [3]. Furthermore, NDs possess special advantages of biocompatibility, easy surface functionalization, high thermal conductivity, and useful optical, physical, and chemical properties, which make it to be an ideal material in a number of applications such as gene and drug delivery vehicles [4], optical contrast agents [5], nanoscale sensors [6], and biological imaging [7]. In these applications, drug molecules or biomolecules could be adsorbed on NDs and were delivered into cells to perform their biological efficacy. Furthermore, NDs were reported to be nontoxic for these biomedical applications. However, the challenge is to understand the reason for the nontoxicity of NDs and the effects of the conjugation of biomolecules with NDs on its toxicity $[8,9]$.

Many efforts have been dedicated to investigate the interactions of NDs with biomolecules for achieving their applications in the biomedical fields. For example, several researchers have used some proteins such as lysozyme, bovine serum albumin (BSA), cytochrome c, and myoglobin to physically adsorb on NDs and investigated their binding properties $[1,8,9]$. The interactions of NDs with red blood cells and NDs surface modification by proteins in blood have been investigated, which confirmed the NDs' safety in organisms [10]. Ermakova et al. used the affinity of ferritin on 
the NDs' surface and the magnetic noise induced by the inner paramagnetic iron to detect ferritin molecules [11]. Usually, the noncovalent affinity is so strong that proteins can easily be captured by NDs through a variety of forces including covalent binding, hydrophobic interaction, electrostatic binding, hydrogen binding, and van der Waals interaction [3], the interactions between NDs and proteins depend on the functioned groups on NDs, the properties of protein, and their reaction conditions. Therefore, understanding the binding mechanism of NDs with proteins is essential for NDs' biotechnological applications.

Human serum albumin (HSA) is the most abundant protein in the circulatory system and possesses specific transportation and deposition of a variety of endogenous ligands, such as fatty acids, and exogenous ligands, such as metal ions, nanoparticles, and a variety of therapeutics drugs $[12,13]$. Interacting with nanoparticles, HSA forms the first layer of the long-lived hard corona on the surface of nanoparticles, which increases their circulatory half-lives in the blood and improves distribution and biocompatibility throughout the body [14]. Several previous reports have investigated the interactions of various nanomaterials with HSA and the induced structure change and stability of proteins [15-17]. Yin et al. investigated the interactions of $\mathrm{Au}$ nanoclusters with three model proteins (HSA, $\gamma$-globulins, and transferrin), and the hydrophobic force between them was proved [18]. Zhang et al. used HSA to modify a gadolinium-fullerene derivative for obtaining a macromolecular magnetic resonance imaging contrast agent [19]. Although most of the earlier studies provided excellent methods to investigate the physicochemical properties of HSA on nanomaterials and extended the applications of nanomaterials, information about the binding mechanism between HSA and NDs and the biological effects of the interaction on NDs have rarely been reported.

In this work, we concentrate on the binding mechanism between NDs and HSA. The influence factors on the interaction between them, the induced conformational changes in protein, and the effect of HSA on the cytotoxicity of NDs were evaluated, which provides a fundamental understanding for the use of NDs as a platform for biomedical applications.

\section{Experimental}

2.1. Chemicals and Reagents. Dispersible nanodiamonds (NDs) with average size about $5-10 \mathrm{~nm}$ were bought from XFNANO Materials Tech Co., Ltd. (Nanjing), which claimed that the purity of carboxylated NDs was higher, that is, $97 \%$ with hydroxyl and carboxyl functional groups. Human serum albumin (HSA) was purchased from SigmaAldrich. MTT (3-(4,5-Dimethylthiazol-2-yl)-2,5-diphenyltetrazolium bromide) assay kit and sodium phosphate buffer (PBS) were purchased from J\&K Scientific Ltd. (China). All other chemicals used in this study were of analytical reagent grade. All aqueous solutions were prepared with high purity water $\left(18.2 \mathrm{M} \Omega \cdot \mathrm{cm}^{-1}\right)$ at ambient temperature.
2.2. Sample Preparation. The stock suspensions of NDs with various concentration were prepared using $0.01 \mathrm{M}$ PBS ( $\mathrm{pH}$ 7.0 ), which were sonicated for $2 \mathrm{~h}$. Because NDs solutions were dispersed well, other further treatment was not performed. The HSA-NDs solution was prepared by adding a certain amount of HSA solution $(2.0 \mathrm{mM})$ to $3.0 \mathrm{~mL}$ different concentrations of NDs solutions in sequence. The mixed solutions were shaken at $180 \mathrm{rpm}$ at $25^{\circ} \mathrm{C}$ for $2 \mathrm{~h}$ and were then used for spectral determination. The HSA-NDs solid samples for Fourier transform infrared (FTIR) detection were separated by centrifugation of HSA-NDs solution with an ultracentrifuge at $12,000 \mathrm{rpm}$ and $4^{\circ} \mathrm{C}$ for $1 \mathrm{~h}$. The precipitation of HSA-NDs was then washed two times with high purity water and was further centrifuged to remove unbound protein molecules. The amount of HSA adsorbed onto NDs could be determined from the difference of HSA concentration before and after addition of the NDs into the solution according to the standard curve of HSA [20], which was described in Supporting Information (Figure S1).

2.3. Analysis of the Interactions between NDs and HSA. Absorption spectra were recorded in a quartz cuvette $\left(1.0 \times 1.0 \mathrm{~cm}^{2}\right)$ using a UV-vis spectrophotometer (TU-1901, Persee, China). Fluorescence analyses were performed on a spectrophotometer (F-7000, Hitachi, Japan) with a quartz cell $\left(1.0 \times 1.0 \mathrm{~cm}^{2}\right)$ at the excitation wavelength of $295 \mathrm{~nm}$ (the slit width is $5 / 5 \mathrm{~nm}$ ). The emission spectra were recorded between 300 and $450 \mathrm{~nm}$ with temperature maintained by circulating bath. Circular dichroism (CD) measurements were performed on a J-810 spectrometer (Tokyo, Japan) with $1.0 \mathrm{~mm}$ path length quartz cell. The CD spectra of $2.0 \mu \mathrm{M}$ HSA with NDs were recorded in the wavelength range of 190-250 nm. Every CD spectrum was obtained from the average of three scans, and the scanning speed was set at $50 \mathrm{~nm} \cdot \mathrm{min}^{-1}$. Dynamic light scattering (DLS) was recorded on a 90 Plus Particle Size Analyzer (Brookhaven Instruments, America) to evaluate the relative hydrodynamic diameter distributions of NDs solutions. In DLS measurements, the NDs and BSA-NDs samples were filtered with $0.45 \mu \mathrm{m}$ membrane filter. Transmission electron microscope (TEM) images of NDs and BSA-NDs were taken on a JEOL-2010 HR transmission electron microscope (JEOL, Japan). Samples were placed on 200 mesh carboncoated copper grids.

2.4. MTT Cell Viability Assay. To evaluate the cytotoxicity of NDs and HSA-NDs complex, the human lung epithelial cell line (BEAS-2B cells) was used and separately incubated with different concentrations of NDs. Because cell culture medium contains $10 \%$ fetal bovine serum (FBS), bovine serum albumin (BSA), which shares structural homology and similar properties with HSA, was used to evaluate the toxicity of NDs in the presence of serum protein. $1 \times 10^{4}$ BEAS-2B cells/well were firstly grown in cell culture medium supplemented with $10 \%$ fetal bovine serum (FBS) for $24 \mathrm{~h}$. After washing twice with $0.01 \mathrm{M}$ PBS, the complete cell culture medium was replaced by stock solutions of NDs or 
BSA-NDs complex, which were prepared to required concentration using cell culture media without FBS $\left(c_{\text {NDs }}: 0,2,4\right.$, $8,16,32$, and $64 \mathrm{mg} \cdot \mathrm{L}^{-1} ; c_{\mathrm{BSA}-\mathrm{ND}}: 6.0 \mu \mathrm{M}$ BSA). The cells in the same culture solution without NDs or BSA-NDs were used as controls. After incubation for $24 \mathrm{~h}$, the morphology of cells was investigated by using the microscope (OLYMPUS IX53). The viability of cells was assessed by MTT assay: the 96-well plates were inverted over a container with a small stack of paper towels and were slightly shaken to remove the culture medium. The cells were washed with PBS for two times and then treated with $100 \mu \mathrm{L}$ fresh culture medium containing $10 \mu \mathrm{L}$ of $5 \mathrm{mg} \cdot \mathrm{mL}^{-1}$ sterile MTT dye (in PBS, $\mathrm{pH}$ 7.0), which were incubated at $37^{\circ} \mathrm{C}$ for $4 \mathrm{~h}$. After that, MTT solution in each well was replaced by $100 \mu \mathrm{L}$ of DMSO. The optical density (OD) for the formazan product was measured at $570 \mathrm{~nm}$, and the percentage of $\mathrm{OD}_{\mathrm{NDs}} / \mathrm{OD}_{\text {control was }}$ represented as the cell viabilities.

\section{Results and Discussion}

3.1. Characterization of NDs and HSA-NDs. The morphology, size, distribution, and the functional groups of NDs and HSA-NDs and their interactions were characterized with transmission electron microscope (TEM), dynamic light scattering (DLS), UV-vis and fourier transform infrared (FTIR) spectroscopies, and circular dichroism (CD) spectrometry.

Figures 1(a) and 1(b) show the TEM images of NDs and HSA adsorbed on NDs. Obviously, NDs exists as small aggregates $(50-150 \mathrm{~nm})$. Every single ND is about $5-10 \mathrm{~nm}$ in size, which is in accordance with the previous reports and that reported by the supplier $(<10 \mathrm{~nm})$, and suggests the excellent dispersion in aqueous solution [21-23]. Although the carboxyl groups on the NDs' surface enhance their dispersion in water, NDs coagulated to form a lot of small aggregates of tens of nanometers, which is probably due to the residual hydrophobic carbon on their surface [21]. After interacting with HSA (Figure 1(b)), NDs shows more dispersed status. Apparent layer of "protein corona" coating the NDs' surface was observed. The aggregates of NDs were broken down to the smaller aggregates. The stability was also further improved. These results suggest that the strong interaction between HSA and NDs occurred and the hydrophilic HSA prevented the aggregation of NDs.

Further evidence of their ground-state interaction comes from DLS measurements. Figures 1(c) and 1(d) show the size distribution histograms of $16.0 \mathrm{mg} \cdot \mathrm{L}^{-1}$ NDs in the absence and presence of HSA, respectively. In the absence of HSA, the hydrodynamic diameters of NDs mainly ranged from 105 to $151 \mathrm{~nm}$ with a mean size of $\sim 126 \mathrm{~nm}$. However, a clear shift of the size was observed in the smaller size region for the HSA-NDs system. The mean diameter decreased to $73 \mathrm{~nm}$ in the presence of HSA (Figure 1(d)). The changing trends of DLS coincide with the results in TEM, which suggests that the interaction between HSA and NDs may be ascribed to the static ground-state association [14].

Figure 2(a) shows the absorption spectra of NDs, HSA, and HSA-NDs. Native HSA exhibits a strong absorption peak at $280 \mathrm{~nm}$, which was mostly attributed to the absorptions of tryptophan (Trp) residues [24]. The NDs solution does not show any obvious absorption peak between $300 \mathrm{~nm}$ and $800 \mathrm{~nm}$. However, in the presence of NDs, the absorption of HSA increases and the absorption peak shifts from $280 \mathrm{~nm}$ to $275 \mathrm{~nm}$, which probably resulted from the change in the polarity around the Trp residues [25]. To investigate the conformation of HSA, circular dichroism (CD) was performed to monitor the secondary structure of HSA in the presence of NDs. As shown in Figure 2(b)) native HSA exhibits two negative absorption bands at $208 \mathrm{~nm}$ and $222 \mathrm{~nm}$, which are assigned to the parallel exactions of the $\pi-\pi^{*}$ transition of peptide and the $n-\pi^{*}$ transition of the carbonyl group, respectively $[14,26]$. The $\alpha$-helix content of HSA is estimated to be $51.7 \%$. In the presence of NDs, the negative peaks slightly rise without any shift in wavelength with increasing NDs concentrations. The $\alpha$-helix contents for HSA-NDs complexes were calculated to be $48.7 \%$ at $8.0 \mathrm{mg} \cdot \mathrm{L}^{-1} \mathrm{NDs}, 46.4 \%$ at $16.0 \mathrm{mg} \cdot \mathrm{L}^{-1} \mathrm{NDs}, 45.4 \%$ at $32.0 \mathrm{mg} \cdot \mathrm{L}^{-1} \mathrm{NDs}$, and $44.1 \%$ at $64.0 \mathrm{mg} \cdot \mathrm{L}^{-1} \mathrm{NDs}$, which are smaller than that of the native HSA. The results suggest that the conformation of HSA on the NDs' surface was slightly altered [27]. FTIR spectra were used to identify the functional groups present in NDs and HSA. As shown in Figure 2(c), the vibrational peaks of NDs at $1780 \mathrm{~cm}^{-1}$ can be assigned to the $\mathrm{C}=\mathrm{O}$ stretching band. The bands near 1641 , 1384 , and $1121 \mathrm{~cm}^{-1}$, can be assigned to $\mathrm{O}-\mathrm{H}$ bending vibration, $\mathrm{O}-\mathrm{H}$ deformation, and $\mathrm{C}-\mathrm{O}$ stretching [28]. The strong $\mathrm{O}-\mathrm{H}$ vibration of $\mathrm{NDs}$ at $3445 \mathrm{~cm}^{-1}$ indicates the presence of hydroxide groups. The spectrum of HSA shows the protein's characteristic amide I at $1661 \mathrm{~cm}^{-1}(\mathrm{C}=\mathrm{O}, \mathrm{C}-\mathrm{N}$ stretching) and amide II bands at $1542 \mathrm{~cm}^{-1}(\mathrm{~N}-\mathrm{H}$ deformation and $\mathrm{C}-\mathrm{N}$ stretching), which are the vibrations of peptide backbones and are conformational-sensitive to ligands or nanoparticles induced structural changes of HSA $[8,10]$. After interacting with NDs, amide I and II bands of HSA were observed on the HSA-NDs complex, indicating that HSA bonded onto the NDs surface [29]. The bands amide I and amide II did not show any shift in the position; however, their relative intensities decreased when compared with those of native HSA, suggesting that the secondary structure of HSA only undergoes slight changes and it retains native conformation during its interaction with NDs [30].

\subsection{Fluorescence Quenching Studies for the Interactions of} NDs with HSA. A fluorescence quenching technique has been effectively and extensively applied to investigate the biding mechanism and conformation of protein interacting with nanomaterials [31]. As shown in Figure 3(a), HSA shows an intrinsic fluorescence at $336 \mathrm{~nm}$ mainly due to the presence of Trp-214 in subdomain IIA [14]. The intrinsic fluorescence of HSA is progressively quenched by the presence of NDs with increasing concentration, which reveals the interacting characteristics of HSA on NDs' surface. Moreover, the maximum emission wavelength $(336 \mathrm{~nm})$ remains unchanged during the interaction, signifying the unaltered tertiary structure of HSA on the surface of NDs. 


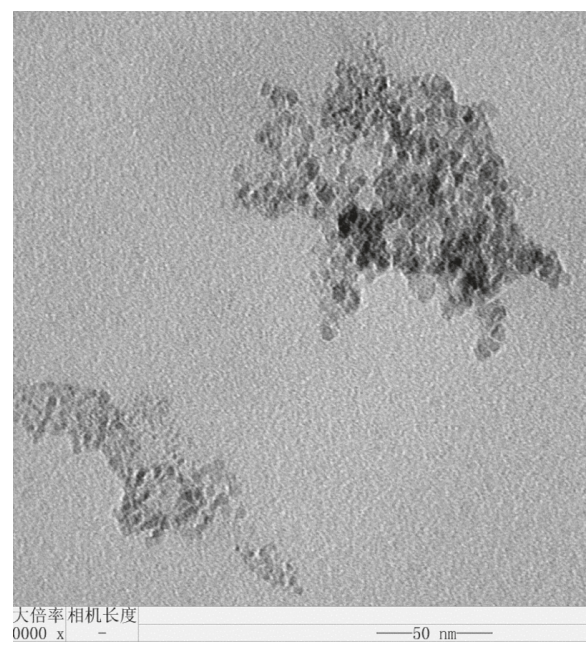

(a)

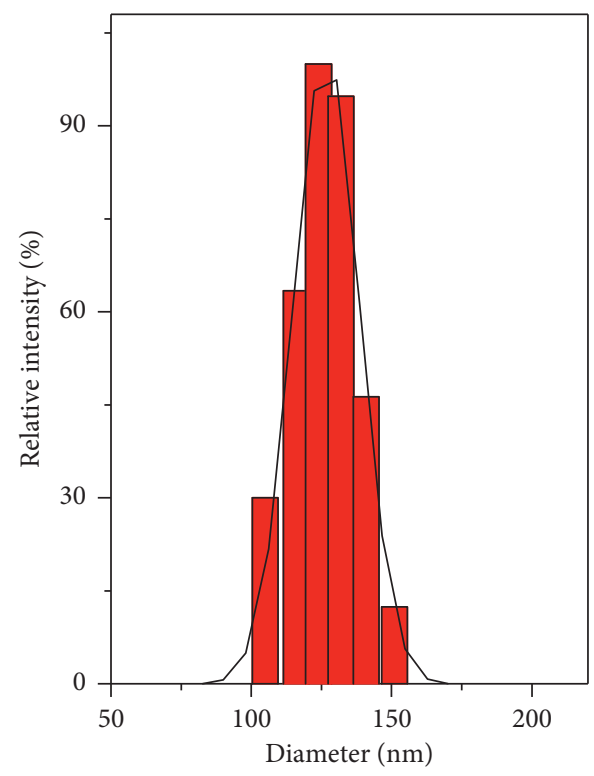

(c)

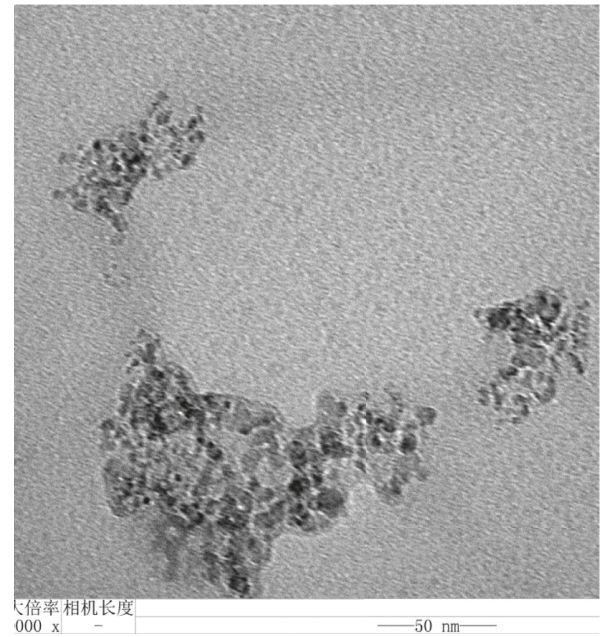

(b)

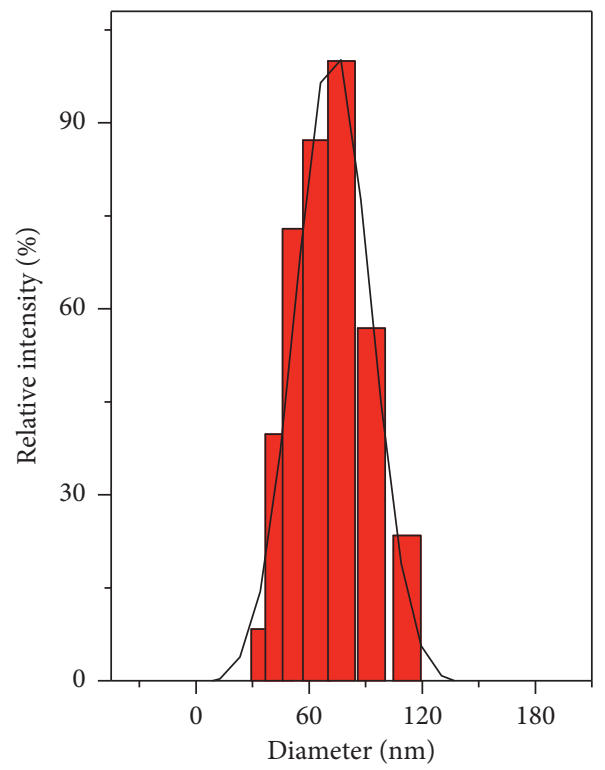

(d)

FIgUre 1: TEM images of NDs (a) and HSA-NDs complex (b). Size distributions of NDs (c) and HSA-NDs complex (d) in 0.01 M PBS determined by DLS. (cNDs $=16.0 \mathrm{mg} \cdot \mathrm{L}-1 ; \mathrm{cHSA}=2.0 \mu \mathrm{M})$.

The similar results at the temperature of 288,303 , and $313 \mathrm{~K}$ were shown in Figure S2.

The fluorescence quenching of protein could be induced by the dynamic quenching and static quenching. Because any shift in $\lambda_{\text {em }}$ was not observed, the quenching may be static quenching resulting from the formation of proteinNDs complex [31]. To clarify the quenching mechanism, fluorescence quenching was analyzed by the Stern-Volmer equation (equation (1)):

$$
\frac{F_{0}}{F}=1+K_{\mathrm{SV}}[Q]=1+k_{\mathrm{q}} \tau_{0}[Q]
$$

where $F_{0}$ and $F$ denote the fluorescence intensities of HSA without and with quencher (NDs), respectively. $K_{\mathrm{sv}}$ is the Stern-Volmer quenching constant, and $k_{\mathrm{q}}$ is the apparent bimolecular quenching rate constant. $\tau_{0}$ is the average lifetime of the protein without quencher, which is about
$5.79 \times 10^{-9} \mathrm{~s}$ for HSA [25]. [Q] is the concentration of the quenchers (NDs). Carboxylated NDs do not have a definite molecular weight. We cannot accurately calculate the molar concentrations of NDs. According to the methods for the carbon nanomaterials in previous reports, weight concentration multiplied by $\lambda$ as pseudomolar concentrations $[32,33]$. For one of the NDs, one diamond crystal cell is comprised by eight carbon atoms and only a carboxyl group is assumed on one diamond crystal cell, so $\lambda$ should be no more than $7.75 \times 10^{-3}$ ). The values of $k_{\mathrm{q}}$ evaluated from the Stern-Volmer plots were shown in Figure 3(b) (298 K) in Table 1. The values of $k_{\mathrm{q}}$ are larger than that of the limiting diffusion rate constant of biomacromolecules $\left(2.0 \times 10^{10} \mathrm{M}^{-1} \cdot \mathrm{s}^{-1}\right)$, which suggests that the quenching mechanism of HSA fluorescence by NDs is mainly due to the formation of static ground-state complex [33]. However, we can find that the $k_{\mathrm{q}}$ values slightly vary and 


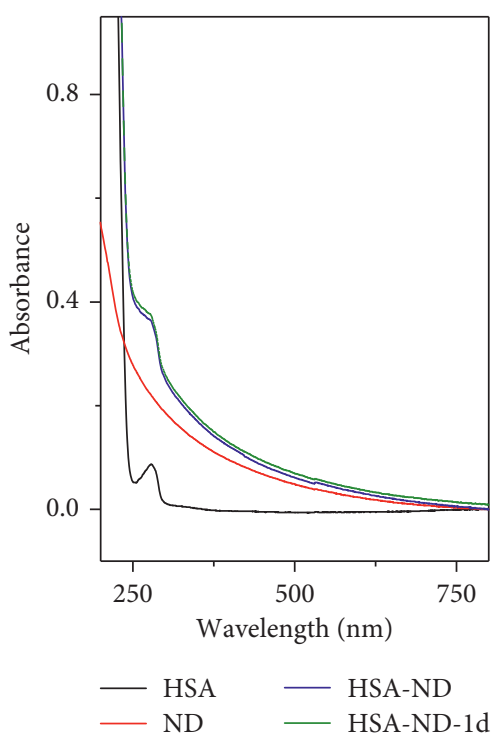

(a)

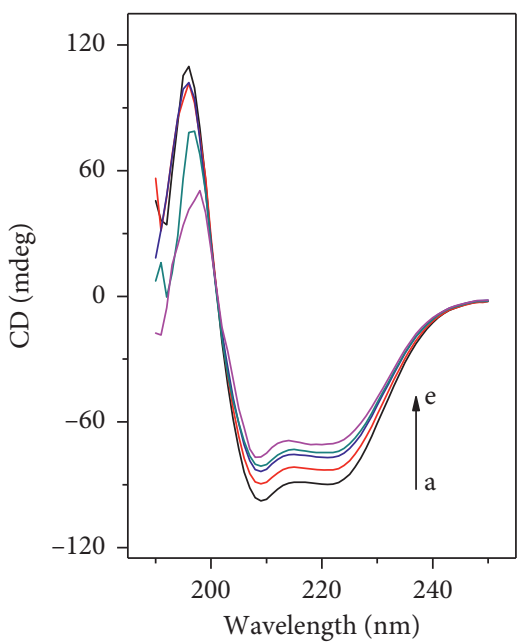

(b)

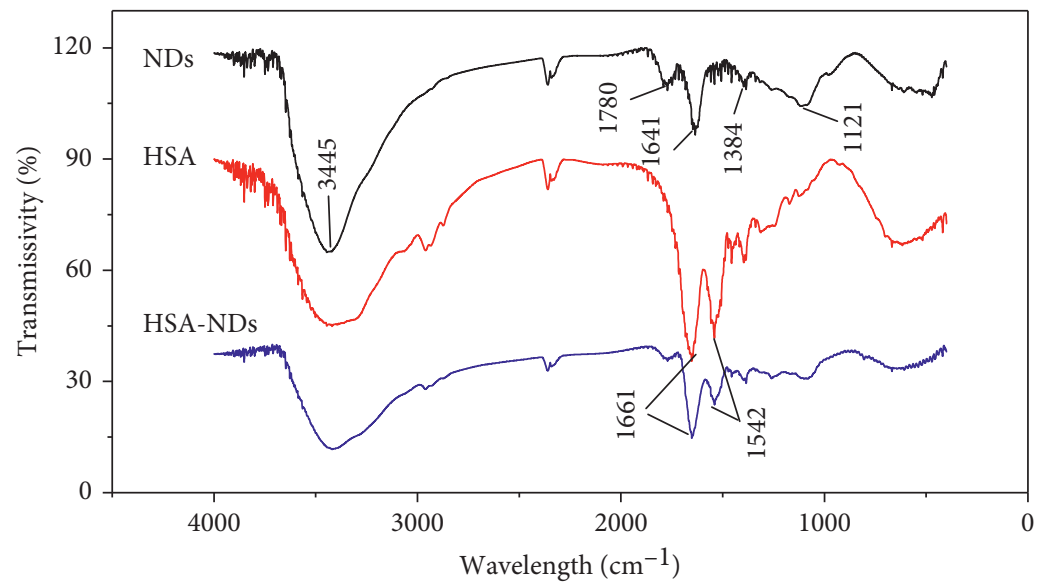

(c)

Figure 2: (a) UV-vis spectra of HSA, NDs, and HSA-NDs (green curve: HSA-NDs system was stored for one day). (b) CD spectra of $1.0 \mu \mathrm{M}$ HSA in $0.01 \mathrm{M}$ PBS (pH 7.4) with various concentration of NDs ( $c_{\mathrm{NDs}}$ from a to e: $0,8.0,16.0,32.0$, and $64.0 \mathrm{mg} \cdot \mathrm{L}^{-1}, T=298 \mathrm{~K}$. (c) FTIR spectra of NDs, HSA, and HSA-NDs complex.

increase with increasing temperature except for $313 \mathrm{~K}$. The results demonstrate that the dynamic collision might participate in quenching processes at high temperature. Therefore, the influence of high temperature on the binding process and protein conformation cannot be ignored.

To obtain the binding constant $\left(K_{\mathrm{b}}\right)$ and binding number $(m)$, the fluorescence quenching data at different temperature were analyzed by the following equation $[32,33]$ :

$$
\log \frac{F_{0}-F}{F}=\log K_{\mathrm{b}}+m \log [Q] .
$$

$K_{\mathrm{b}}$ and $m$ values obtained from the linear plots (Figure 3(c)) were summarized in Table $1 . K_{\mathrm{b}}$ and $m$ decreased with increasing temperature, suggesting that high temperature decreased the binding sites as well as the stability of the HSA-NDs.

The interacting forces for the interactions between proteins and ligands can be inferred from the thermodynamic parameters. The thermodynamic parameters such as enthalpy change $\left(\Delta H^{\circ}\right)$, entropy change $\left(\Delta S^{\circ}\right)$, and the free energy change $\left(\Delta G^{\circ}\right)$ can be calculated from the $K_{\mathrm{b}}$ at different temperatures according to the equations (equations (3) and (4)) [34]. $\Delta H^{\circ}$ is considered to be independent in a small temperature range.

$$
\begin{aligned}
\ln K_{\mathrm{b}} & =\frac{-\Delta H^{\circ}}{R T}+\frac{\Delta S}{R}=\ln \left(\frac{K}{\lambda^{m}}\right), \\
\Delta^{G^{\circ}} & =\Delta^{H^{\circ}}-T \Delta^{S^{\circ}},
\end{aligned}
$$

where $R$ is the gas constant $8.314 \mathrm{~J} \cdot \mathrm{mol}^{-1} \cdot \mathrm{K}^{-1}$ and $T$ is temperature $(\mathrm{K}) . K$ represents the equilibrium constant calculated just using the mass concentration $\left(K_{b}=K / \lambda^{m}\right)$. From the fitted line in Figure 3(d) and the obtained values of $\Delta H^{\circ}, \Delta S^{\circ}$, and $\Delta G^{\circ}$ in Table 1 , negative values of $\Delta G^{\circ}$ manifest that the interaction between HSA and NDs is spontaneous. Both $\Delta H^{\circ}$ and $\Delta S^{\circ}$ are negative values, indicating that hydrogen 


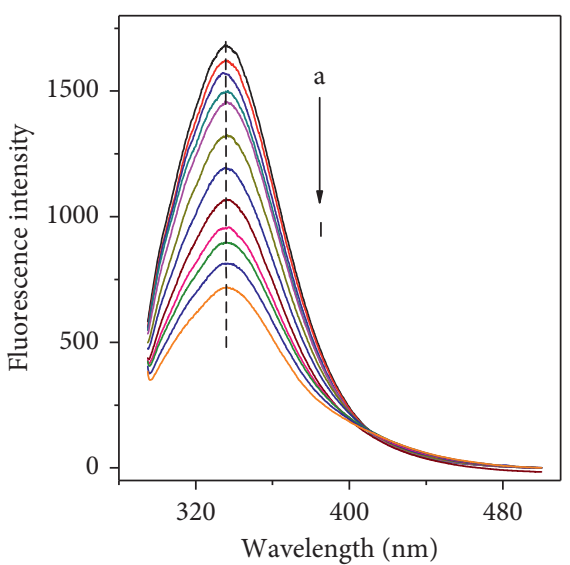

(a)

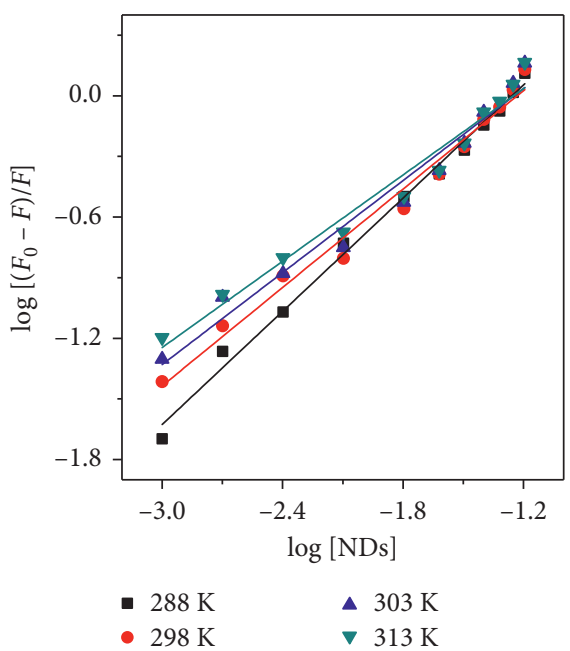

(c)

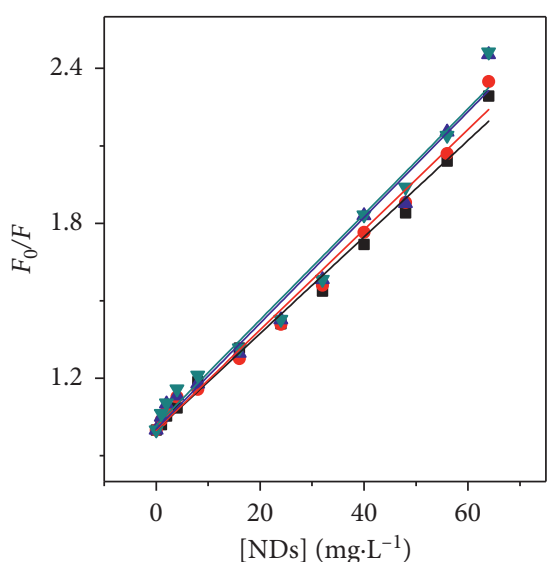

- $288 \mathrm{~K} \Delta 303 \mathrm{~K}$

- $298 \mathrm{~K}$

จ $313 \mathrm{~K}$

(b)

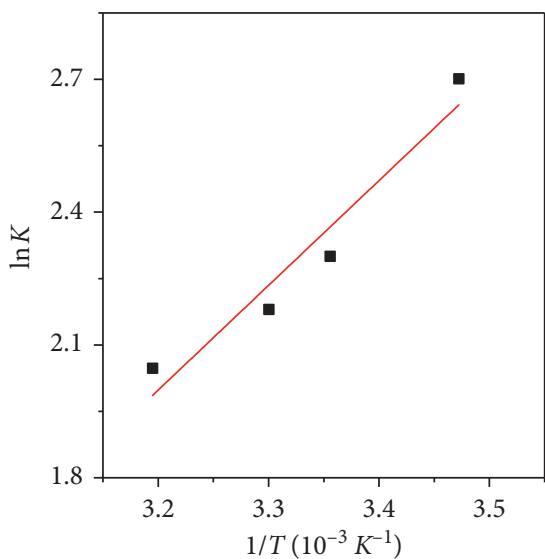

(d)

FiguRE 3: (a) Fluorescence quenching of $6.0 \mu \mathrm{M}$ HSA observed with increasing NDs concentrations in $0.01 \mathrm{M}$ PBS (pH 7.4; T=298 K; $\lambda_{\text {ex }}=285 \mathrm{~nm} ; c_{\mathrm{NDs}}$ from a to $\mathrm{l}$ are $0,1.0,2.0,4.0,8.0,16.0,24.0,32.0,40.0,48.0,56.0$, and $64.0 \mathrm{mg} \cdot \mathrm{L}^{-1}$ ). (b) The Stern-Volmer plots for the fluorescence quenching of HSA with NDs. (c) Plots of log $\left[F_{0}-F / F\right] v s$. log [NDs] at different temperature. (d) van't Hoff plot for the HSAND system.

TABLE 1: Various quenching constants, binding parameters, and thermodynamic parameters of HSA upon interaction with NDs at different temperatures.

\begin{tabular}{lccccccc}
\hline Sample & $T(\mathrm{~K})$ & $K_{\mathrm{q}} \times 10^{9}\left(\mathrm{~L} \cdot \mathrm{mol}^{-1} \cdot \mathrm{s}^{-1}\right)$ & $m$ & $K_{\mathrm{b}}\left(\mathrm{L} \cdot \mathrm{mol}^{-1}\right)$ & $\Delta G\left(\mathrm{~kJ} \cdot \mathrm{mol}^{-1}\right)$ & $\Delta H\left(\mathrm{~kJ} \cdot \mathrm{mol}^{-1}\right)$ & $\Delta S\left(\mathrm{~J} \cdot \mathrm{mol}^{-1} \cdot \mathrm{K}^{-1}\right)$ \\
\hline \multirow{3}{*}{ HSA-ND } & 288 & $3.23 / \lambda$ & 0.93 & $14.9 / \lambda^{0.93}$ & $-6.33+2.22 \ln \lambda$ & & \\
& 298 & $3.35 / \lambda$ & 0.81 & $9.98 / \lambda^{0.81}$ & $-5.87+2.01 \ln \lambda$ & -19.67 & $-46.31-8.31 \ln \lambda^{m}$ \\
& 303 & $3.54 / \lambda$ & 0.76 & $8.85 / \lambda^{0.76}$ & $-5.64+1.91 \ln \lambda$ & \\
\hline
\end{tabular}

bonding and van der Waals forces play the major role in the binding process between HSA and NDs [32]. At physiological pH 7.0, the carboxyl groups were deprotonated and induced the negatively charged surfaces of NDs [23]. Furthermore, the isoelectric point (pI) of HSA is $\sim 4.7$ and thus HSA shows negatively charged surface at pH 7.0 [14]. Therefore, the electrostatic interactions and hydrophobic interaction between HSA and carboxylated NDs can be eliminated, which coincides with the results of thermodynamic analysis.

3.3. Cytotoxicity for the NDs and HSA-NDs Systems. Previous studies have demonstrated that NDs are practically nontoxic and have good biocompatibility in cell-based assays and animal models; thus, NDs have been widely used 

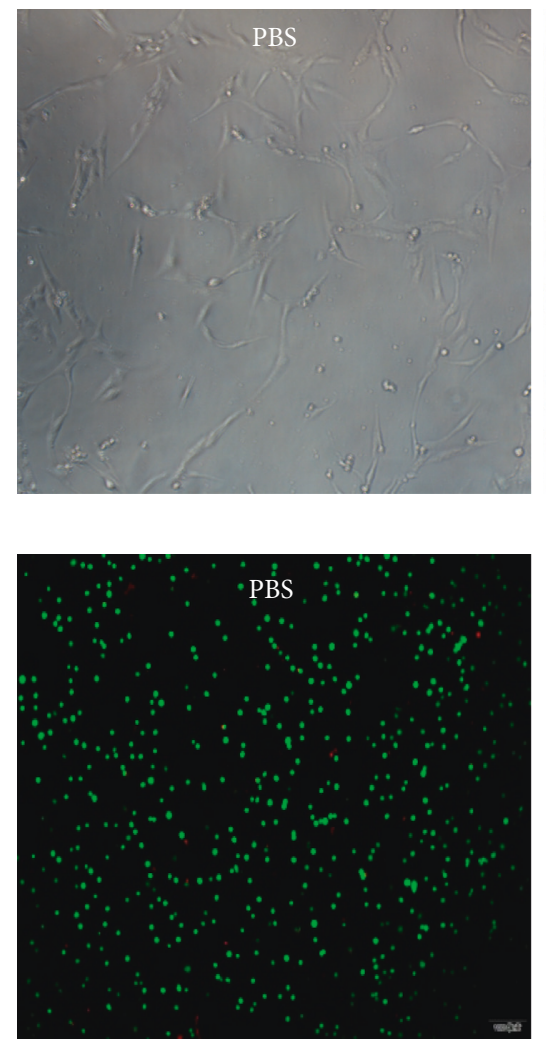
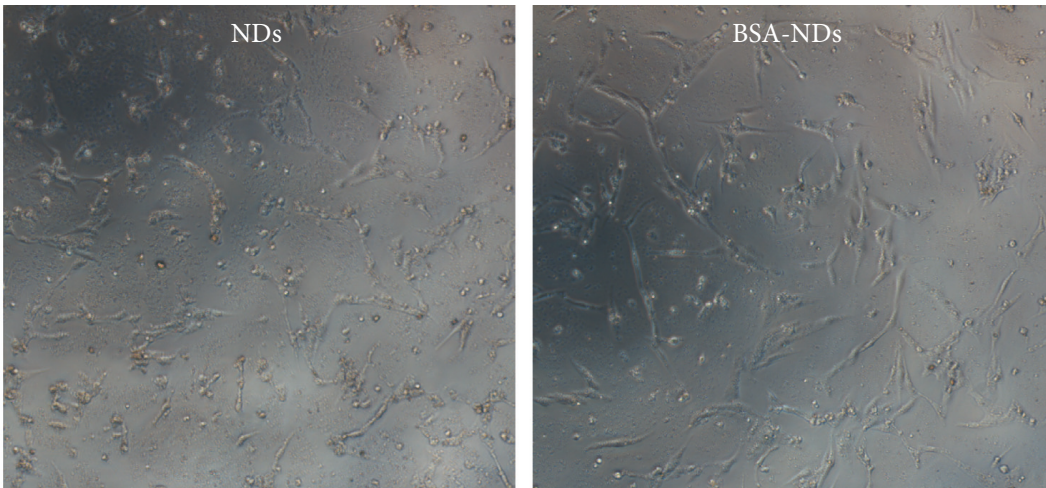

(a)
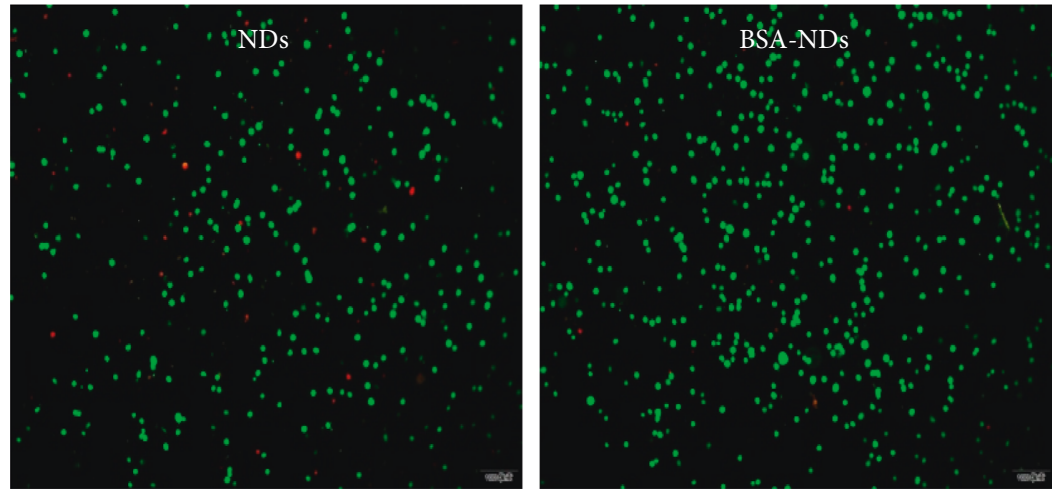

(b)

FIgURE 4: (a) Optical microscope images of BEAS-2B cells cultured with PBS, NDs without serum protein, and BSA-NDs. (b) Viable cells were stained green with calcein $\mathrm{AM}$ and dead cells were stained red with ethidium homodimer-1 ( $\left.c_{\mathrm{PBS}}: 0.01 \mathrm{M} ; c_{\mathrm{NDs}}: 8 \mathrm{mg} \cdot \mathrm{L}^{-1} ; c_{\mathrm{BSA}}: 6.0 \mu \mathrm{M}\right)$.

for delivery agents for anticancer drugs and circulating proteins $[35,36]$. For further evaluation of the potential toxicity of NDs and the cellular uptake of HSA-NDs, a human lung epithelial cell line (BEAS-2B) was carried out by a series of in vitro experiments. BEAS-2B cells were firstly incubated in serum-free medium with different concentrations of NDs for $24 \mathrm{~h}$, and then, the viability of the cell line was evaluated using the MTT methods. As shown in Figure 4(a), there is no obvious difference for BEAS-2B cells in cell-culture medium and in PBS without serum. When BEAS-2B cells incubated with NDs in serumfree medium, NDs appeared on the bright-field microphotographs as brown dots, which aggregated inside the cells or on the cell surface. Cell death and changes in cell morphology were observed with high concentration of NDs. Similar trends were observed in the fluorescencebased live/dead assays. Obvious cell death occurred in the presence of $8 \mathrm{mg} \cdot \mathrm{L}^{-1} \mathrm{NDs}$ without BSA. In the BSA-ND system, dead cells were obviously reduced containing $6.0 \mu \mu \mathrm{M}$ BSA. As shown in Figure 5, the viability of BEAS$2 \mathrm{~B}$ cells decreased to $\sim 54 \%$ when they are exposed to $32 \mathrm{mg} /$ $\mathrm{L}$ NDs. However, in the presence of $6.0 \mu \mathrm{M}$ BSA, the viabilities of BEAS-2B cells are obviously higher than those at the same concentration of NDs. Apparently the serumdependent and concentration-dependent cytotoxicity of NDs were observed, which confirmed that the NDs were either nontoxic or toxic to BEAS-2B cells depending on

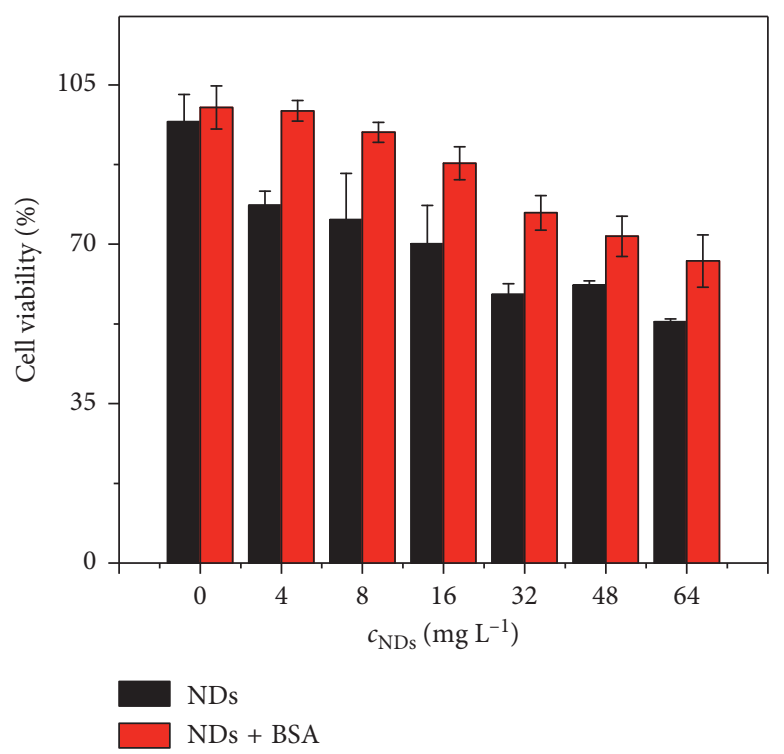

Figure 5: Viability of BEAS-2B cells exposed to various concentrations of NDs for $24 \mathrm{~h}\left(c_{\mathrm{NDs}}: 0,4,8,16,32\right.$, and $64 \mathrm{mg} \cdot \mathrm{L}^{-1} ; c_{\mathrm{BSA}}$ : $6.0 \mu \mathrm{M} ; n=5)$.

whether the serum existed in the culture medium [20]. The results confirmed that the cytotoxicity of NDs was eliminated after NDs interacting with BSA. 


\section{Conclusion}

This work investigated the binding affinity of NDs towards HSA and the cytotoxicity of NDs toward BEAS-2B cells. NDs exhibited high binding activity toward HSA mainly by the hydrophobic force. Based on the interactions, the "protein corona" obviously formed and the coated HSA on NDs surface basically retained its native conformation although with slight decrease of the $\alpha$-helical content. NDs showed obvious cytotoxicity in serum-free medium; however, the toxicity of NDs toward BEAS-2B cells could be mitigated by the interaction between NDs and proteins. Therefore, when we use NDs as drug delivery vehicles, NDs should be performed in complete cell culture medium, which contains $10 \%$ fetal bovine serum.

\section{Data Availability}

The data used to support the findings of this study are available from the corresponding author upon request.

\section{Conflicts of Interest}

The authors declare that there are no conflicts of interest.

\section{Acknowledgments}

This work was supported by the National Natural Science Foundation of China (21405019, 91643113, 21637004), the cooperative project between Fuyang municipal government and Fuyang Normal University (XDHX201701, XDHX201704), the Natural Science Foundation of Anhui Province (1708085MB43), the Natural Science Foundation of Higher Education Institutions in Anhui province (KJ2019ZD37, KJ2018ZD035), the Innovation team of modern analytical technologies (kytd201701), the State Key Laboratory of Analytical Chemistry for Life Science (SKLACLS1712), and the Anhui provincial teaching team program (2017jxtd025).

\section{Supplementary Materials}

Figure S1: (A) the fluorescence spectra of HSA in 0.01 M PBS with various concentrations (curves from bottom to top: 0.4 , $0.8,1.6,2.4,3.2,4.0,4.8$, and $5.6 \mu \mathrm{M})$ at different temperatures $\left(T=298 \mathrm{~K} ; \lambda_{\mathrm{ex}}=285 \mathrm{~nm}\right)$; (B) the calibration curve between fluorescence intensity and HSA concentration. Figure S2: fluorescence quenching of $6.0 \mathrm{mM}$ HSA observed with increasing NDs concentrations in $0.01 \mathrm{M} \mathrm{PBS}$ at $288 \mathrm{~K}$ (A), $303 \mathrm{~K}(\mathrm{~B})$, and $313 \mathrm{~K}(\mathrm{C})\left(\mathrm{pH} 7.4 ; \lambda_{\mathrm{ex}}=285 \mathrm{~nm}\right.$; cNDs from a to $l$ are $0,1.0,2.0,4.0,8.0,16.0,24.0,32.0,40.0,48.0$, 56.0 , and $64.0 \mathrm{mg} \cdot \mathrm{L}^{-1}$ ). (Supplementary Materials)

\section{References}

[1] C.-L. Lin, C.-H. Lin, H.-C. Chang, and M.-C. Su, "Protein attachment on nanodiamonds," The Journal of Physical Chemistry A, vol. 119, no. 28, pp. 7704-7711, 2015.

[2] Q. Feng, X. Zhao, Y. Guo, M. Liu, and P. Wang, "Stochastic DNA walker for electrochemical biosensing sensitized with gold nanocages@graphene nanoribbons," Biosensors and Bioelectronics, vol. 108, pp. 97-102, 2018.

[3] L. Pishkar, S. Taheri, S. S. Makarem et al., "Studies on the interaction between nanodiamond and human hemoglobin by surface tension measurement and spectroscopy methods," Journal of Biomolecular Structure and Dynamics, vol. 5, no. 283, pp. 603-615, 2017.

[4] K. Zhang, Q. Zhao, S. Qin et al., "Nanodiamonds conjugated upconversion nanoparticles for bio-imaging and drug delivery," Journal of Colloid and Interface Science, vol. 537, pp. 316-324, 2019.

[5] K. van der Laan, M. Hasani, T. Zheng, and R. Schirhagl, "Nanodiamonds for in vivo applications," Small, vol. 14, no. 19, Article ID 1703838, 2018.

[6] R. Schirhagl, K. Chang, M. Loretz, and C. L. Degen, "Nitrogen-vacancy centers in diamond: nanoscale sensors for physics and biology," Annual Review of Physical Chemistry, vol. 65, no. 1, pp. 83-105, 2014.

[7] K. Bray, L. Cheung, K. R. Hossain, I. Aharonovich, S. M. Valenzuela, and O. Shimoni, "Versatile multicolor nanodiamond probes for intracellular imaging and targeted labeling," Journal of Materials Chemistry B, vol. 6, no. 19, pp. 3078-3084, 2018.

[8] E. Perevedentseva, P.-J. Cai, Y.-C. Chiu, and C.-L. Cheng, "Characterizing protein activities on the lysozyme and nanodiamond complex prepared for bio applications," Langmuir, vol. 27, no. 3, pp. 1085-1091, 2011.

[9] X. L. Kong, L. C. L. Huang, C.-M. Hsu, W.-H. Chen, C.-C. Han, and H.-C. Chang, "High-affinity capture of proteins by diamond nanoparticles for mass spectrometric analysis," Analytical Chemistry, vol. 77, no. 1, pp. 259-265, 2005.

[10] L.-W. Tsai, Y.-C. Lin, E. Perevedentseva, A. Lugovtsov, A. Priezzhev, and C.-L. Cheng, "Nanodiamonds for medical applications: interaction with blood in vitro and in vivo," International Journal of Molecular Sciences, vol. 17, no. 7, p. 1111, 2016.

[11] A. Ermakova, G. Pramanik, J.-M. Cai et al., "Detection of a few metallo-protein molecules using color centers in nanodiamonds," Nano Letters, vol. 13, no. 7, pp. 3305-3309, 2013.

[12] A. Lacroix, T. G. W. Edwardson, M. A. Hancock, M. D. Dore, and H. F. Sleiman, "Development of DNA nanostructures for high-affinity binding to human serum albumin," Journal of the American Chemical Society, vol. 139, no. 21, pp. 7355$7362,2017$.

[13] N. Jafari, R. Ahmed, M. Gloyd, J. Bloomfield, P. BritzMcKibbin, and G. Melacini, "Allosteric sensing of fatty acid binding by NMR: application to human serum albumin," Journal of Medicinal Chemistry, vol. 59, no. 16, pp. 7457-7465, 2016.

[14] A. Bhattacharya, S. Das, and T. K. Mukherjee, "Insights into the thermodynamics of polymer nanodot-human serum albumin association: a spectroscopic and calorimetric approach," Langmuir, vol. 32, no. 46, pp. 12067-12077, 2016.

[15] Y. Y. Foo, M. Z. Kabir, V. Periasamy, S. N. A. Malek, and S. Tayyab, "Spectroscopic studies on the interaction of green synthesized-gold nanoparticles with human serum albumin," Journal of Molecular Liquids, vol. 265, pp. 105-113, 2018.

[16] M.-Y. Li, C.-Q. Xiao, Z.-Q. Xu et al., "Role of surface charge on the interaction between carbon nanodots and human serum albumin," Spectrochimica Acta Part A: Molecular and Biomolecular Spectroscopy, vol. 204, pp. 484-494, 2018.

[17] C. Carnovale, G. Bryant, R. Shukla, and V. Bansal, "Impact of nanogold morphology on interactions with human serum," 
Physical Chemistry Chemical Physics, vol. 20, no. 46, pp. 29558-29565, 2018.

[18] M.-M. Yin, P. Dong, W.-Q. Chen et al., "Thermodynamics and mechanisms of the interactions between ultrasmall fluorescent gold nanoclusters and human serum albumin, $\gamma$-globulins, and transferrin: a spectroscopic approach," Langmuir, vol. 33, no. 21, pp. 5108-5116, 2017.

[19] Y. Zhang, T. Zou, M. Guan et al., "Synergistic effect of human serum albumin and fullerene on Gd-DO3A for tumor-targeting imaging," ACS Applied Materials \& Interfaces, vol. 8, no. 18, pp. 11246-11254, 2016.

[20] J. Li, Y. Zhu, W. Li, X. Zhang, Y. Peng, and Q. Huang, "Nanodiamonds as intracellular transporters of chemotherapeutic drug," Biomaterials, vol. 31, no. 32, pp. 8410-8418, 2010.

[21] M.-C. Kim, D. Lee, S. H. Jeong, S.-Y. Lee, and E. Kang, "Nanodiamond-gold nanocomposites with the peroxidaselike oxidative catalytic activity," ACS Applied Materials of Interfaces, vol. 8, no. 50, pp. 34317-34326, 2016.

[22] R. A. Shimkunas, E. Robinson, R. Lam et al., "Nanodiamondinsulin complexes as $\mathrm{pH}$-dependent protein delivery vehicles," Biomaterials, vol. 30, no. 29, pp. 5720-5728, 2009.

[23] D. G. Lim, J. H. Jung, H. W. Ko, E. Kang, and S. H. Jeong, "Paclitaxel-nanodiamond nanocomplexes enhance aqueous dispersibility and drug retention in cells," ACS Applied Materials \& Interfaces, vol. 8, no. 36, pp. 23558-23567, 2016.

[24] S. Li, X. C. Zhao, Y. M. Mo, P. T. Cummings, and W. T. Heller, "Human serum albumin interactions with $\mathrm{C}_{60}$ fullerene studied by spectroscopy, small-angle neutron scattering, and molecular dynamics simulations," Journal of Nanoparticle Research, vol. 15, no. 7, pp. 1-11, 2013.

[25] G. Rabbani, M. H. Baig, E. J. Lee, W.-K. Cho, J. Y. Ma, and I. Choi, "Biophysical study on the interaction between eperisone hydrochloride and human serum albumin using spectroscopic, calorimetric, and molecular docking analyses," Molecular Pharmaceutics, vol. 14, no. 5, pp. 1656-1665, 2017.

[26] H. Wu, P. Wang, X. Hu, Z. Dai, and X. Zou, "Site-selective probe for investigating the asynchronous unfolding of domains in bovine serum albumin," Talanta, vol. 84 , no. 3 , pp. 881-886, 2011.

[27] J. Kuchlyan, N. Kundu, D. Banik, A. Roy, and N. Sarkar, "Spectroscopy and fluorescence lifetime imaging microscopy to probe the interaction of bovine serum albumin with graphene oxide," Langmuir, vol. 31, no. 51, pp. 13793-13801, 2015.

[28] D.-K. Lee, S. V. Kim, A. N. Limansubroto et al., "Nanodiamond-gutta percha composite biomaterials for root canal therapy," ACS Nano, vol. 9, no. 11, pp. 11490-11501, 2015.

[29] H.-D. Wang, C. H. Niu, Q. Yang, and I. Badea, "Study on protein conformation and adsorption behaviors in nanodiamond particle-protein complexes," Nanotechnology, vol. 22, no. 14, Article ID 145703, 2011.

[30] A. S. Sharma and M. Ilanchelian, "Comprehensive multispectroscopic analysis on the interaction and corona formation of human serum albumin with Gold/Silver alloy nanoparticles," The Journal of Physical Chemistry B, vol. 119, no. 30, pp. 9461-9476, 2015.

[31] I. Jha and P. Venkatesu, "Deciphering the interactions of bromelain with carbon nanotubes: role of protein as well as carboxylated multiwalled carbon nanotubes in a complexation mechanism," The Journal of Physical Chemistry C, vol. 120, no. 28, pp. 15436-15445, 2016.

[32] X. Zhao, R. Liu, Z. Chi, Y. Teng, and P. Qin, "New insights into the behavior of bovine serum albumin adsorbed onto carbon nanotubes: comprehensive spectroscopic studies," The Journal of Physical Chemistry B, vol. 114, no. 16, pp. 5625-5631, 2010.

[33] H. Wu, M. Chen, M. Shang et al., "Insights into the binding behavior of bovine serum albumin to black carbon nanoparticles and induced cytotoxicity," Spectrochimica Acta Part A: Molecular and Biomolecular Spectroscopy, vol. 200, pp. 51-57, 2018.

[34] P. D. Ross and S. Subramanian, "Thermodynamics of protein association reactions: forces contributing to stability," Biochemistry, vol. 20, no. 11, pp. 3096-3102, 1981.

[35] K. E. Zakrzewska, A. Samluk, M. Wierzbicki et al., "Analysis of the cytotoxicity of carbon-based nanoparticles, diamond and graphite, in human glioblastoma and hepatoma cell lines," PLoS One, vol. 10, Article ID e0122579, 2015.

[36] X. Hu, X. Li, M. Yin et al., "Nanodiamonds mediate oral delivery of proteins for stem cell activation and intestinal remodeling in Drosophila," ACS Applied Materials \& Interfaces, vol. 9, no. 22, pp. 18575-18583, 2017. 

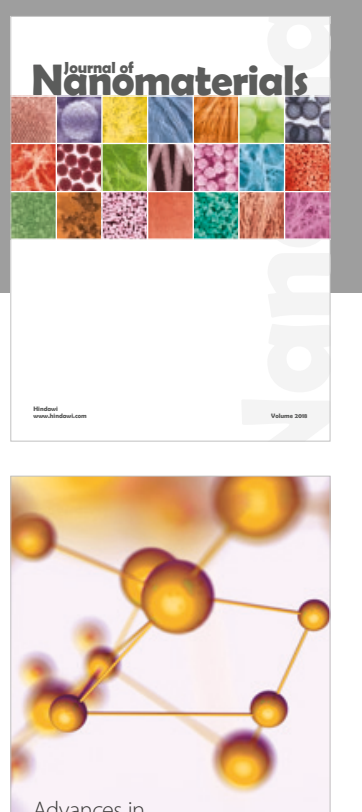

Physical Chemistry
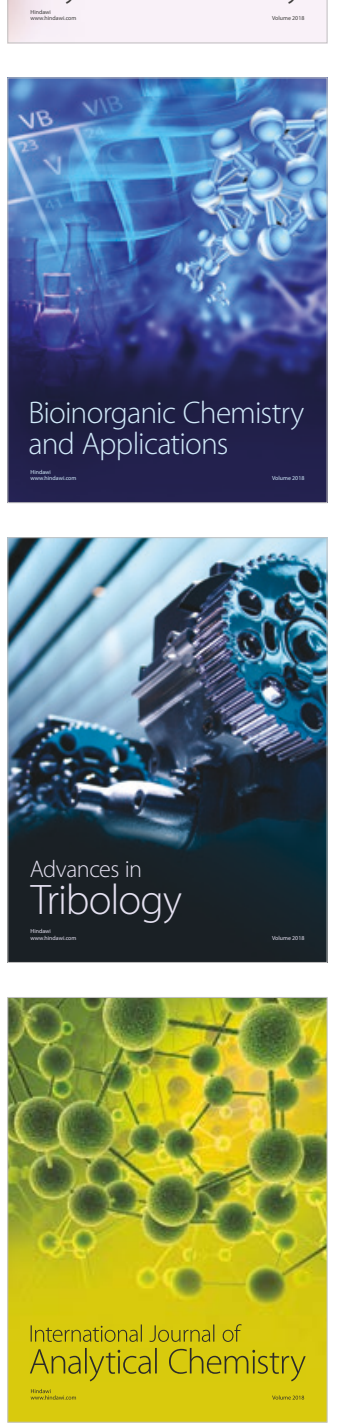

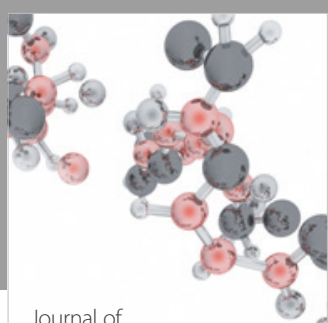

Analytical Methods

in Chemistry

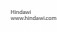

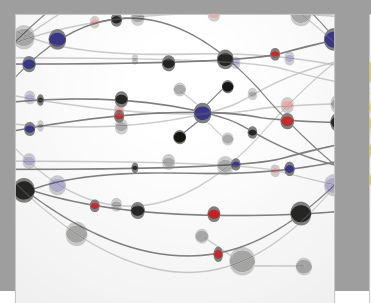

The Scientific World Journal

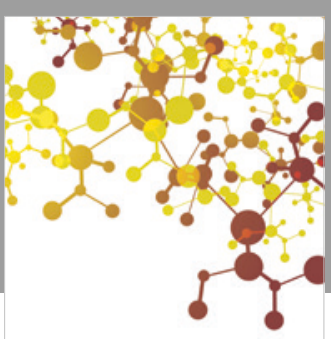

Journal of

Applied Chemistry
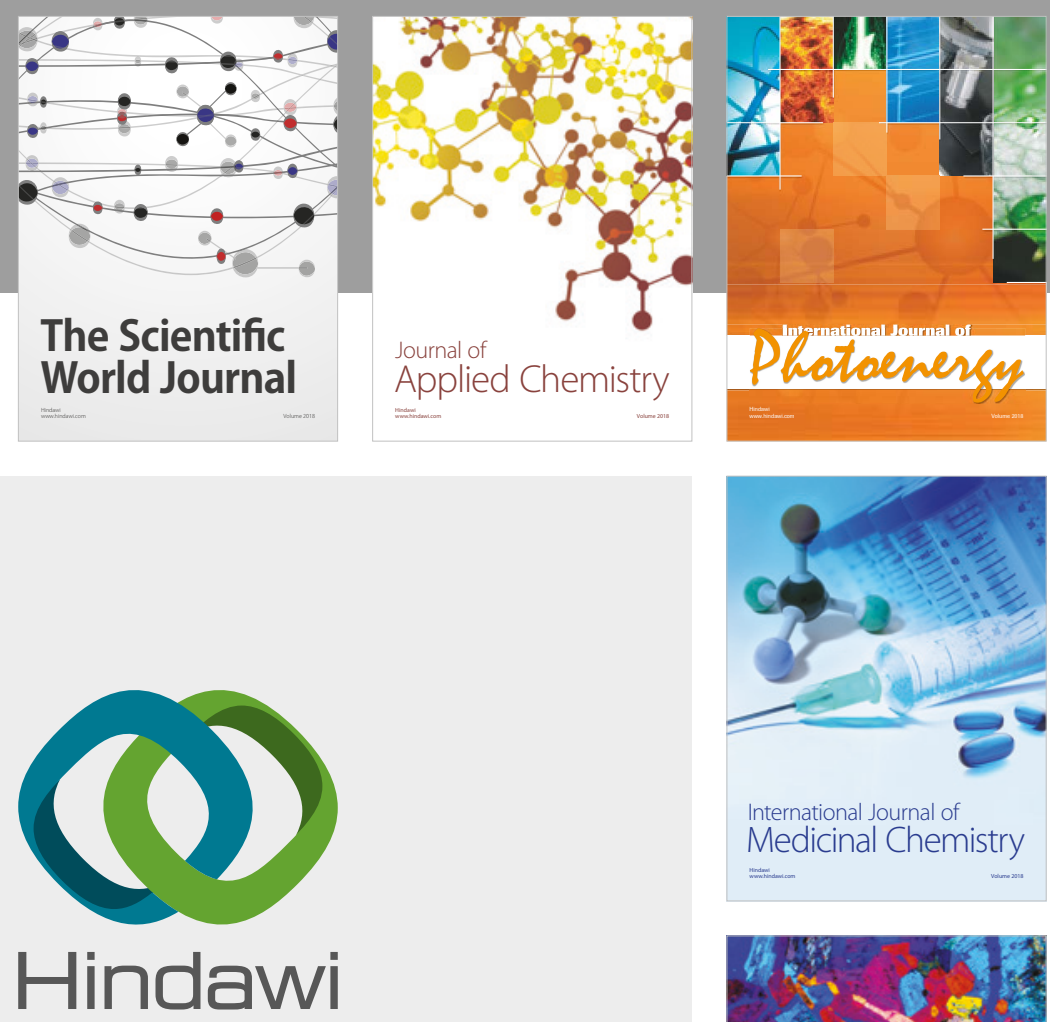

Submit your manuscripts at

www.hindawi.com
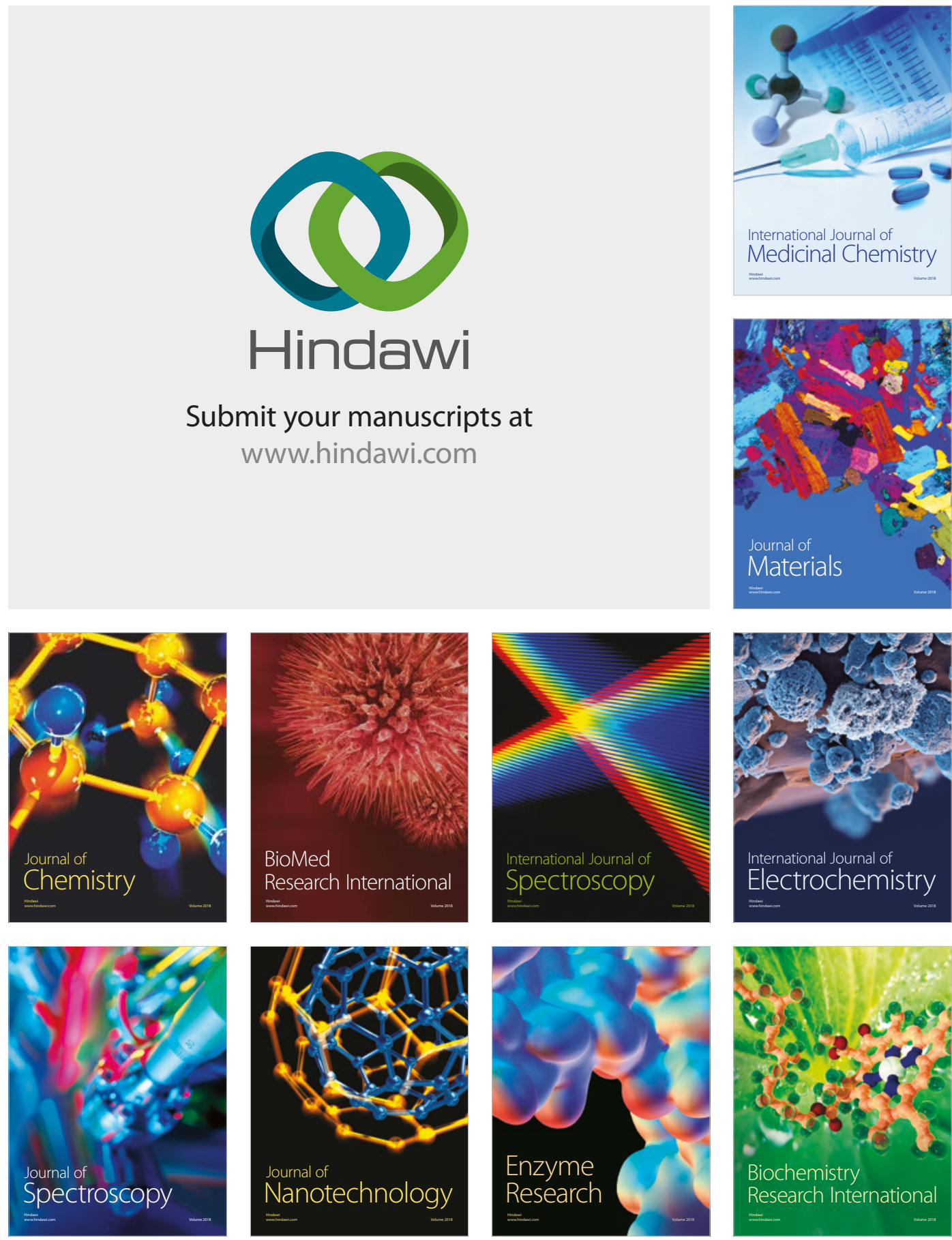
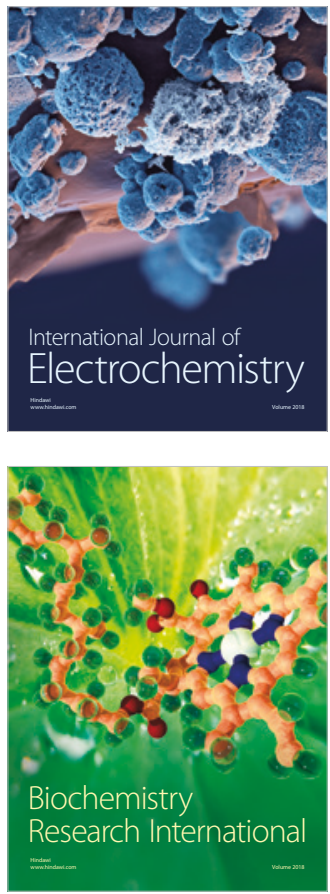